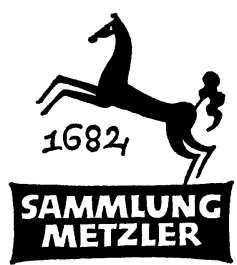

REALIEN ZUR LITERATUR

ABT. D:

LITERATURGESCHICHTE 
HORST NÄGELE

\section{J. P. Jacobsen}

MCMLXXIII

J. B. METZLERSCHE VERLAGSBUCHHANDLUNG

STUTTGART 
ISBN 978-3-476-10117-4

ISBN 978-3-476-03835-7 (eBook)

DOI 10.1007/978-3-476-03835-7

M 117

(C) Springer-Verlag GmbH Deutschland 1973

Ursprünglich erschienen bei J. B. Metzlersche Verlagsbuchhandlung und Carl Ernst Poeschel Verlag GmbH in Stuttgart 1973 


\section{VORBEMERKUNG}

Entsprechend den Zielsetzungen der iSammlung Metzler sollen durch die nachstehende Darstellung erste Hilfen gegeben werden für eine wissenschaftliche Beschäftigung mit dem Phänomen ,J. P. Jacobsen'. Im Interesse größtmöglicher Effizienz werden kritische Bemerkungen zur Anlage und Ausstattung des Bändchens wie die Mitteilung weiteren Materials begrüßt.

Die vorliegende Erstauflage verdankt wesentliche Hinweise Hanns Grössel (Köln), Jan E. Janssen (Århus), Hans Peter Johannsen (Flensburg), Jörgen Knudsen (Egtved), Sven Möller Kristensen (Kopenhagen), Jörgen Ottosen (Kopenhagen), Villy Sörensen (Hvidovre), Reiner Steinweg (Frankfurt am Main), Hanne Marie und Werner Svendsen (Kopenhagen), Jörn Vosmar (Århus), Werner Welzig (Wien), dem Historischen Seminar der Universität Kiel (Jörn-Peter Leppien), dem Leiter der Handschriftenabteilung der Königlichen Bibliothek in Kopenhagen (Tue Gad) sowie der Leiterin des Heimatmuseums von Thisted (Jette Kjær); zu keiner Antwort ist es gekommen auf Rückfragen bei Ruth Berlau (Berlin), dem Bertolt-Brecht-Archiv in Berlin wie auch bei Frederik Nielsen (Dänemark).

Gedankt sei den Direktoren und Mitarbeitern einer Reihe von Bibliotheken, deren Dienste bei der Zusammenstellung des Materials in Anspruch genommen wurden. Der Hans Reitzels Forlag A/S, Kopenhagen, ermöglichte ein Einsichtnehmen in `OmMG (s. u., S. VIII) bereits vor dem Erscheinen (26. 7. 1972).

Kiel, im September 1972

HORST NÄGELE 


\section{INHALT}

I. BIbLIOGRAPHIEN ．．．．．．．．．．．．．． I I

II. TeXTe . . . . . . . . . . . . . . . . . . . . . . . . I I

r. Handschriften . . . . . . . . . . . . . . I

2. Werkausgaben ............... 2

3. Notizen, Briefwechsel . . . . . . . . . . . . . . 3

III. Die literarischen Phänomene: CEuvre . . . . . . . . 4

r. Romane . . . . . . . . . . . . . . . . . . . . . . 4

"Fru Marie Grubbe« . . . . . . . . . . . . . . . . . 4

"Niels Lyhne« .. . . . . . . . . . . . . . . I0

2. Novellen und Skizzen. . . . . . . . . . . . . . 16

»Mogens" . . . . . . . . . . . . . . . . . . . . 17

$\gg$ Et Skud i Taagen« . . . . . . . . . . . . . . . . . 20

"To Verdener« . . . . . . . . . . . . . . . . . . . 21

"Fra Skitsebogen« ("Der burde have været Roser«) . . . 22

»Pesten i Bergamo« . . . . . . . . . . . 23

"Fru Fönss« . . . . . . . . . . . . . . . . . . 23

»Udlændinge $«$. . . . . . . . . . . . . . . . . . 24

„Kormark og Stengerde«. . . . . . . . . . . . 25

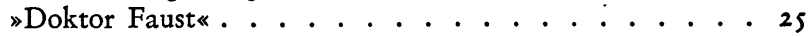

Weitere Erzählfragmente, Prosaentwürfe, Gedankensplitter . . . . . . . . . . . . . . . . . . . 25

3. Gedichte und poetische Versuche . . . . . . . . . . . 26

4. Populärwissenschafliche Abhandlungen . . . . . 30

IV. ZUR FRAGE NACH DER ,LITERARISCHEN INDIVIDUALITÄT J. P. JACOBSEN $^{6}$. . . . . . . . . . . . . . . 32

I. Der Begriff, literarische Individualität ${ }^{\circ}$. . . . . . . . 32

2. Das, Individunm J. P. Jacobsen" . . . . . . . . . 36

Chronologische Úbersicht zu ,J. P. Jacobsen (1847-85) 38

Biographien ............. . 4I

V.Zur Frage Nach DEM, IDEOlogischen Kontext' . . . $4 \mathrm{I}$

r. Der Begriff ,gesellschafliches Wesen von Kunst ${ }^{\circ}$. . . . 4I

2. Historisch-politische Aspekte . . . . . . . . . . . 44 Manifestationen eines neuen politischen ,Sprachspiels in Dänemark . . . . . . . . . . . . . 46

VI. Zu einer ,TeXtcharakteristik' . . . . . . . . . . . 49

VII. ZUR FRAGE NACH ,REFLEXEN' IN DER DEUTSCHEN LITERATUR SI

Dokumente zur Jacobsen-Mode . . . . . . . . 65

Register : : : . . . . . . . . . . . . 70 


\section{Siglen und Chiffren}

b

Bd

bd

DS

dt.

Edda

GRM

Hg. (hg.)

$\mathrm{Jb}$.

Jg.

Jh.

Kh

Kritik

Lit.

neugedr.

OL

OmMG

OmNL

PMLA

Slg

SV

Zs.

Ztg (wesentlich) bearbeitete Auflage (die Chiffre steht, hochgestellt, vor hochgestellten Auflagenummern)

Band

bind/Bind (dänisch für ,Band')

Danske Studier (Kopenhagen und Odense)

deutsch

Edda. Nordisk tiddsskrift for litteraturforskning (Oslo)

Germanisch-Romanische Monatsschrift

Herausgeber (herausgegeben)

Jahrbuch

Jahrgang

Jahrhundert

Köbenhavn/Kopenhagen

Kritik. Tidsskrift for litteratur - forskning - undervisning, (Kopenhagen und Ārhus)

Literatur

neugedruckt

Orbis Litterarum. International Review of Literary Studies (Kopenhagen und Odense)

Omkring Fru Marie Grubbe, udgivet af J. Ottosen (Kopenhagen: Reitzels Værkserie 1972) = eine Dokumentation zu "Fru Marie Grubbe «

Omkring Niels Lyhne, udgivet af N. Barfoed (Kopenhagen: Reitzels Værkserie 1970) = eine Dokumentation zu »Niels Lyhne«

Publications of the Modern Language Association of America

Sammlung

J. P. Jacobsen: Samlede Værker = historisch-textkritische Werkausgabe durch M. Borup (siehe unten, S. 2 f.) Zeitschrift

Zeitung 


\section{ABgekÜ̈ZT ZITIERTE LITERATUR}

v. ANDERSEN $(1905)=$ V. A.: Jens Peter Jacobsen, in Samtiden 17 (1905), pp. 410-423, 449-463 (neugedruckt in: V. A., Litteraturbilleder. Anden Samling, Kh 1907, 2 1946).

v. ANDERSEN (1925) = V. A.: J. P. Jacobsen, in: Carl Petersen og V. A., Illustreret dansk Litteraturhistorie, bd 4, Kh 1925, pp. 206-225.

s. ARestad $(1965)=$ S. A.: J. P. Jacobsens »Niels Lyhne«, in: Scandinavian Studies. Essays Presented to Dr. Henry Goddard Leach on the Occasion of His Eighty-fifth Birthday, The University of Washington Press, Seattle 1965, pp. 202-212.

L. BAER (1939) = L. B.: Rilke and Jens Peter Jacobsen, in:PMLA 54 (1939), Pp. 900-933, II33-II80.

F. J. BILleskov JANSEN (1943) = F.J.B.J.: Aandsvidenskaber og Digtning, in: Dansk Daad, bd 3 , Kh r943, pp. I23-177.

E. BRANDES (1899) = E. B.: Forord in: Breve fra J. P. Jacobsen, Kh I899, ${ }^{2}$ I 899, b $^{3}$ I $925,{ }^{4}$ I 968 (s. u. S. 3 f.).

G. BRANDES $(1883)=$ G. B.: Det moderne Gjennembruds Mænd. En Række Portræter, Kh I883, ${ }^{2}$ I 89 I (neugedr. in: G. B., Samlede Skrifter, bd 3, Kh 1900, ${ }^{2}$ 1919; sowie in: G. B., Essays, udvalgt af W. Svendsen, Kh 1963 (Gyldendals Ugleböger); sowie in: G. B., Danske Digterportrætter, udg. af S. Möller Kristensen, Kh 1964, 2 I 966 (Gyldendals Ugleböger).

G. BRANDES (I885) = G. B.: J. P. Jacobsen (1885), in: G. B., Essays. Danske Personligheder, Kh 1889 (neugedr. in: G. B., Samlede Skrifter, bd 3, Kh 1900, ${ }^{2}$ 1919. Deutsch in: G. BRANDEs, Brandes, Gesammelte Schriften. Deutsche Originalausgabe, $\mathrm{Bd}$ 2, München: Langen 1902; und: G. BRANDES, Gesammelte Schriften, Bd 2, Dresden: Reissner 1924; siehe im übrigen die bibliographische Liste in: B. Nolin, Den gode Europén. Studier i Georg Brandes' idéutveckling 1871-1893 med speciell hänsyn till hans förhållande till tysk, engelsk, slavisk och fransk litteratur, Stockholm i965, pp. 437-439.

TH. BREDSDORFF (1904) = Th. B. [der ältere]: Dansk Digtning I8701900. J. P. Jacobsen, in: Den danske Höjskole 4 (1904), Pp. I6I195, 350-384.

H. BRIX (r926) $=$ H. B.: Danmarks Digtere, Kh $1926,{ }^{2}$ 1944, ${ }^{3}$ I95 I, ${ }^{4} 1962$ (Minerva-bögerne).

c. BURChARDT $(1947)=$ C. B.: J. P. Jacobsen, in: C. B., J. P. Jacobsen og andre essays, Oslo I947, pp. 7-50.

G. Christensen (I9IO) = G. Chr.: J. P. Jacobsen, Kh I910, ${ }^{2}$ I 923. N. FERLOV (1952) = N. F.: Om Niels Lyhne, in: J. P. Jacobsen, Niels Lyhne (udg. af Dansklærerforeningen), Kh. I952, ${ }^{2}$ I 962. 
AA. KNUDSEN (1950) = Aa. K.: J. P. Jacobsen $\mathrm{i}$ hans digtning, Kh I950.

w. H. котAS $(1925)=$ W. H. K.: Die skandinav. Literatur der Gegenwart seit 1870, 1925, S. 22-27.

A. LINCK (I9II) = A. L.: J. P. Jacobsen. Et Levnetslöb. Paa Grundlag af Digterens efterladte Papirer, Kh I9II, b2 1926, ${ }^{3}$ I947 (s. u. S. $4 \mathrm{I}$ ).

S. MÖLLER KRISTENSEN $(1938)$ = S. M. K.: Æstetiske Studier i dansk Fiktionsprosa 1870-1900, Kh I938, b2 I955 (= Impressionismen i dansk prosa 1870-1900. Med et tillæg om stiludviklingen efter år 1900) [mit Lit.].

s. MÖLLER KRISTENSEN $(1963)=$ S. M. K.: Danmarks litteratur fra I 870 til nutiden, in: M. Bröndsted og S. M. K., Danmarks litteratur, Kh 1963 (ständig Neudrucke).

F. NIELSEN (1953) = F. N.: J. P. Jacobsen. Digteren og mennesket. En litterær undersögelse, Kh 1953 [mit kurzer Zusammenfassung in engl. Spr.], ${ }^{2}$ I 968 [unverändert, doch ohne die Zusammenfassung in engl. Spr.].

J. OTTOSEN (1968) = J. O.: J. P. Jacobsens "Mogens«, Kh 1968.

H. STANGERUP $(1966)=$ H. St.: J. P. Jacobsen, in: Dansk Litteraturhistorie, Kh: Politikens Forlag 1966, pp. 58-95.

E. Thomsen $(1935)=$ E. Th.: J. P. Jacobsen, in: E. Th., Dansk Litteratur efter 1870. Med Sideblik til det övrige Norden, Kh 1935, 21962.

B. TIGERSCHIÖLD (1945) = B. T.: J. P. Jacobsen och hans roman Niels Lyhne, Göteborg r945.

K. WENTZEL (1968) = K. W.: J. P. Jacobsen. En hank til en virkelighed. Om Hervert Sperring, Niels Lyhne og Arabesk, in: Aa. Henriksen, H. Therkildsen og K. Wentzel (Hg.), Den erindrende Faun, Kh 1968, pp. 52-75. 